Annals of Mathematics, 155 (2002), 209-234

\title{
Embeddedness of minimal surfaces with total boundary curvature at most $4 \rho$
}

\author{
By Tobias Ekholm, Brian White, and Daniel Wienholtz*
}

\begin{abstract}
This paper proves that classical minimal surfaces of arbitrary topological type with total boundary curvature at most $4 \pi$ must be smoothly embedded. Related results are proved for varifolds and for soap film surfaces.

In a celebrated paper [N3] of 1973, Nitsche proved that if $\Gamma$ is an analytic simple closed curve in $\mathbb{R}^{3}$ with total curvature at most $4 \pi$, then $\Gamma$ bounds exactly one minimal disk $M$. Furthermore, that disk is smoothly immersed: it has no branch points, either in the interior or at the boundary. His analysis left open the following questions:

(i) Must $M$ in fact be embedded?

(ii) If $\Gamma$ bounds other minimal surfaces, must they also be free of branch points, or even be smoothly embedded?

In this paper, we show the answer to both questions is "yes," even for curves in $\mathbb{R}^{N}$.

Regarding (ii), we give an example of such a $\Gamma$ in $\mathbb{R}^{3}$ that does indeed bound at least two other minimal surfaces, namely Möbius strips. We conjecture that any such $\Gamma$ can bound at most two Möbius strips, and no surfaces of other topological types. (See $\S 5$.)

Before stating our main result, we review some terminology. The total curvature of a polygonal curve is the sum of the exterior angles at the vertices. For an arbitrary continuous curve, the total curvature is the supremum of the total curvatures of inscribed polygonal curves. This definition, suggested by

*The authors were supported in part by: (Ekholm) Stiftelsen för internationalisering av forskning och högre utbildning, (White) National Science Foundation Grant DMS-9803493 and the Guggenheim Foundation, and (Wienholtz) the Deutsche Forschungsgemeinschaft.

1991 Mathematics Subject Classification. Primary 53A10, secondary 49 F10.

Key words and phrases. Minimal surfaces, total curvature.
\end{abstract}


Fox, was introduced and analyzed in a paper by Milnor [Mi]. In case the curve is piecewise smooth, this definition agrees with the classical one: the integral of the norm of the curvature vector with respect to arclength plus the sum of the exterior angles at the vertices.

Any bounded curve of finite total curvature is rectifiable (i.e., has finite arclength; see $\S 10.1)$. If $\gamma$ is an arclength parametrization of such a curve, then the right and left derivatives $T^{+}$and $T_{-}$of $\gamma$ exist and are unit vectors at each interior point $(\S 10.3,10.4)$. The exterior angle of the curve at any interior point is defined to be the angle between $T^{+}$and $T_{-}$.

Finally, if $M$ is a surface in $\mathbb{R}^{N}$ and $p$ is a point in $\mathbb{R}^{N}$, the density of $M$ at $p$ is

$$
\Theta(M, p)=\lim _{r \rightarrow 0} \frac{\operatorname{area}(M \cap \mathbf{B}(p, r))}{\pi r^{2}}
$$

provided this limit exists, where $\mathbf{B}(p, r)$ is the ball of radius $r$ centered at $p$.

We can now state our main theorem:

Theorem $(2.1,3.2,4.1)$. Let $\Gamma$ be a simple closed curve in $\mathbb{R}^{N}$ with total curvature $\leq 4 \pi$. Let $M$ be a minimal surface with boundary $\Gamma$. Then $M$ is embedded up to and including the boundary, with no interior branch points. Furthermore, at each boundary point $p$ with exterior angle $\theta$, the density of $M$ at $p$ is either $\frac{1}{2}-\frac{\theta}{2 \pi}$ or $\frac{1}{2}+\frac{\theta}{2 \pi}$. At a cusp (i.e., where $\theta=\pi$ ), the density of $M$ is 0 unless $\Gamma$ is contained in a plane.

These density bounds imply, via standard regularity arguments, that for piecewise $C^{1, \alpha}$ boundaries, $M$ has a well-defined tangent plane at every noncusp point, and that in a neighborhood of the point, $M$ is a graph over the tangent plane. In particular, if the boundary is smooth, then the surface is smoothly embedded (and therefore has no branch points.)

Here and throughout the paper, "simple closed curve" means the image of a circle under a continuous one-to-one map. "Minimal surface" means classical minimal surface, that is, a continuous and (possibly branched) conformal harmonic map of a compact 2-manifold with boundary into $\mathbb{R}^{N}$ such that the restriction to the boundary is one-to-one. In Sections 7 and 8, we consider more general minimal varieties. For instance, we prove, roughly speaking, that there are no singular minimal varieties with total boundary curvature $\leq 3 \pi$, and we give a new proof that soap films cannot form on nonclosed wires with total curvature $\leq 2 \pi[\mathrm{DW}]$.

The proof that the interior of $M$ is embedded and unbranched (Theorem 2.1) is an immediate consequence of the following three facts:

(i) The density of a minimal surface $M$ at a point $p \notin \partial M$ is $\leq$ the density at $p$ of the cone subtended by $\partial M$. 
(ii) The density at $p$ of the cone in (i) is at most $1 /(2 \pi)$ times the total curvature of $\partial M$.

(iii) The density of $M$ at any branch point or self-intersection point $p \notin \partial M$ is at least 2 .

Fact (i) follows from an extension of the familiar monotonicity formula for minimal surfaces. We give two proofs of (ii), one based on the Gauss-Bonnet formula and the other on integral geometry. Fact (iii) is well-known.

Analogous facts hold for boundary points.

After completing this paper, we were informed that fact (i) had been discovered by M. Gromov; see Theorem 8.2.A in $[\mathrm{Gr}]$. We have retained our proof to keep the paper self-contained and because (for certain extensions of our main theorem) it is necessary to check that fact (i) holds under very weak assumptions on boundary behavior. Also, deducing it from the extended monotonicity formula as we have done perhaps makes the result more geometrically intuitive (although the proofs are essentially the same).

The $4 \pi$ in our results is in all respects quite sharp. For example, regarding embeddedness, Almgren and Thurston [AT] and then Hubbard [Hub] (in a simpler way) showed that for each $\varepsilon>0$ and $n<\infty$, there is a smooth simple closed curve $\Gamma$ in $\mathbb{R}^{3}$ (knotted or unknotted as desired) with total curvature $<4 \pi+\varepsilon$ such that $\Gamma$ bounds within its convex hull no embedded surface of genus $\leq n$. Such a curve necessarily bounds nonembedded minimal surfaces of each genus $\leq n$. To see this, let $M_{g}$ be a least-area surface of genus $\leq g$ (which exists according to Douglas's theorem: see [Sh1], [DHKW, 11.5], [TT2], or $[\mathrm{J}]$ ). For $g<n, M_{g}$ necessarily has self-intersections, which implies that its area may be reduced by cut-and-paste surgery that adds a one-handle. Thus area $\left(M_{g+1}\right)<\operatorname{area}\left(M_{g}\right)$, which implies that the genus of $M_{g+1}$ is greater than the genus of $M_{g}$. Since this holds for all $g \leq n$, in fact we must have $\operatorname{genus}\left(M_{g}\right)=g$ for every $g \leq n+1$.

The $4 \pi$ is also sharp regarding branch points. For if $F: \mathbf{B}(0,1) \subset \mathbf{C} \rightarrow \mathbb{R}^{N}$ is a minimal surface with a simple branch point at 0 (i.e., if $\partial F / \partial z: \mathbf{B}(0,1) \rightarrow$ $\mathbf{C}^{N}$ has order of vanishing 1 at $\left.z=0\right)$, then $F$ restricted to a small ball $\mathbf{B}(0, r)$ will have total boundary curvature only slightly more than $4 \pi$. Of course if $F \mid \partial \mathbf{B}(0, r)$ is not embedded, one can perturb $\mathbf{B}(0, r)$ slightly to get a domain $\Omega \subset \mathbf{C}$ for which $F \mid \partial \Omega$ is embedded (except in the degenerate case when the image of $F$ is contained in a 2-plane). Incidentally, $F \mid \Omega$ is not the only minimal disk bounded by $F \mid \partial \Omega$, since the least area disk with this boundary cannot have branch points $([\mathrm{O} 1],[\mathrm{Gu} 1],[\mathrm{Alt}])$. Thus this example also shows that the $4 \pi$ in Nitsche's uniqueness theorem is sharp. Indeed, Böhme [Boh] gave examples of curves in $\mathbb{R}^{3}$ with total curvature $4 \pi+\varepsilon$ (with $\varepsilon$ arbitrarily small) that bound many minimal disks, some with branch points. 


\section{History}

In this section, we survey other theorems asserting that certain classical minimal surfaces are embedded and/or free of branch points.

The first embeddedness theorem is due to Radó [Ra2, III.10], who proved that if $\Gamma \subset \mathbb{R}^{N}$ has a one-to-one projection onto the boundary of a convex planar region $R$, then any minimal disk bounded by $\Gamma$ is the graph of a smooth function over $R$. In particular, it is smoothly embedded. If in addition $N=$ 3 , then there is only one disk and there are no minimal varieties of other topological types [Me].

Concerning branch points, Osserman [O1] proved that an area-minimizing minimal surface in $\mathbb{R}^{3}$ cannot have true interior branch points. Gulliver [Gu1] and Alt [Alt] proved that it cannot have false branch points, either. Gulliver and Lesley $[\mathrm{GuL}]$ proved that it cannot have boundary branch points if the boundary curve is analytic. It is not known if the Gulliver-Lesley theorem holds for smooth (say $C^{\infty}$ ) boundaries. (See [Wi] for a partial result.) However, Hildebrandt $[\mathrm{H}]$ and Heinz-Tomi $[\mathrm{HT}]$ (with subsequent improvements by other authors $[\mathrm{N} 2],[\mathrm{K}]$, [War], [L]) proved that a minimal surface in $\mathbb{R}^{N}$ is as differentiable as its boundary at the boundary itself and that boundary branch points must be isolated.

Federer [Fed], refining a theorem of Wirtinger, proved that area minimizing surfaces in $\mathbb{R}^{N}$ with $N \geq 4$ can have interior branch points. Specifically, he proved that any complex variety in $\mathbf{C}^{N} \cong \mathbb{R}^{2 N}$ is absolutely area minimizing: it has less area than any other oriented surface, regardless of topological type. Thus, for example, $z \mapsto\left(z^{2}, z^{3}\right)$ gives a branched, area minimizing map from the unit disk in $\mathbf{C} \cong \mathbb{R}^{2}$ to $\mathbf{C}^{2} \cong \mathbb{R}^{4}$. Nevertheless, White [Wh5] showed that the Gulliver-Lesley theorem remains true in $\mathbb{R}^{N}$. Here the analyticity is essential: there is an example of an absolutely area minimizing surface in $\mathbb{R}^{4}$ with a boundary branch point at a $C^{\infty}$ boundary. This example is obtained from the example due to Gulliver [Gu2] in $\mathbb{R}^{6}$ (as stated in [Wh5]) by restricting to the first four coordinates.

Gulliver and Spruck $[\mathrm{GuS}]$ proved by a continuity argument that if $\Gamma \subset \mathbb{R}^{3}$ has total curvature $\leq 4 \pi$ and if it is extreme (i.e., it lies on the boundary of a convex set), then the minimal disk (unique by Nitsche's result) is in fact embedded. Later Meeks and Yau proved that such a $\Gamma$ does not bound any other minimal varieties [MY2]. Gulliver and Spruck conjectured that either condition alone (curvature $\leq 4 \pi$ or extreme) suffices for existence of a smooth embedded minimal disk. Shortly afterward, three sets of authors proved (in very different ways) that the extremality condition does indeed suffice. Tomi and Tromba [TT1] proved by a more sophisticated continuity argument that an extreme $\Gamma \subset \mathbb{R}^{3}$ bounds a (possibly unstable) smooth embedded minimal 
disk. Almgren and Simon $[\mathrm{AS}]$ proved that among all smooth embedded disks bounded by an extreme $\Gamma$, there is one of least area. Meeks and Yau [MY1] proved the strongest result: the Douglas-Radó disk (the least area disk among all disks bounded by $\Gamma$ ) must in fact be smoothly embedded. Of course our results show that the $4 \pi$ condition also suffices for embeddedness.

We have already mentioned Nitsche's uniqueness theorem. The first step in Nitsche's proof was to exclude branch points in any minimal disk with total boundary curvature $\leq 4 \pi$; this he did using the Gauss-Bonnet formula. (The Gauss-Bonnet formula for branched surfaces is due to Sasaki [Sa], though he made a mistake concerning boundary branch points. The mistake was pointed out and corrected by Nitsche [N1], [N4, §380].) Schneider [Sch] also gave a proof (using ideas of Radó [Ra2, III]) excluding interior branch points for curves of total curvature $\leq 4 \pi$. Schneider's proof does not require any smoothness (beyond continuity) of the boundary curve. In general, the Nitsche and Schneider arguments can be used to bound the total number of branch points (weighted according to the order of branching) of a minimal surface in terms of the genus and total boundary curvature. Our approach gives a bound on the maximum density (and therefore order of branching) at a single point in terms of the total boundary curvature but independent of genus.

\section{Total curvature and densities of cones}

THEOREM 1.1. Suppose $\Gamma$ is a closed curve in $\mathbb{R}^{N}$ and that $p$ is a point not in $\Gamma$. Then

$$
\operatorname{Length}\left(\Pi_{p} \Gamma\right) \leq \text { TotalCurvature }(\Gamma),
$$

where $\Pi_{p}$ is the radial projection to the unit sphere centered at $p$ :

$$
\begin{aligned}
& \Pi_{p}: \mathbb{R}^{N} \backslash\{p\} \rightarrow \partial \mathbf{B}(p, 1) ; \\
& \Pi_{p}(x)=p+\frac{x-p}{|x-p|} .
\end{aligned}
$$

Equivalently,

$$
\Theta\left(\text { Cone }_{p} \Gamma, p\right) \leq \frac{\text { TotalCurvature }(\Gamma)}{2 \pi},
$$

where

$$
\mathrm{Cone}_{p} \Gamma=\{p+t(x-p): x \in \Gamma, 0 \leq t \leq 1\} .
$$

Proof 1 (using Gauss-Bonnet). Consider first the case when $\Gamma$ is smooth. Since the formula is not affected by dilations about $p$, we may assume that $\Gamma$ lies outside the unit ball $\mathbf{B}(p, 1)$ centered at $p$. Let

$$
A=\left(\text { Cone }_{p} \Gamma\right) \backslash \mathbf{B}(p, 1)
$$


be the annular region between $\Gamma$ and $\Pi_{p} \Gamma$. By the Gauss-Bonnet theorem,

$$
\int_{\partial A} \mathbf{k} \cdot \mathbf{n} d s+\int_{A} K=2 \pi \chi(A)
$$

where $\mathbf{k}$ is the curvature vector of the curve $\partial A$ in $\mathbb{R}^{N}, \mathbf{n}$ is the exterior normal of $A, K$ is the scalar curvature of $A$, and $\chi(A)$ is the Euler characteristic of $A$. For $A$ we have $K=0$ and $\chi(A)=0$. Thus

$$
\begin{aligned}
0 & =\int_{\partial A} \mathbf{k} \cdot \mathbf{n} d s \\
& =\int_{\Pi_{p} \Gamma} \mathbf{k} \cdot \mathbf{n} d s+\int_{\Gamma} \mathbf{k} \cdot \mathbf{n} d s
\end{aligned}
$$

The first integral in $(*)$ is equal to the length of $\Pi_{p} \Gamma$, and the second is bounded in absolute value by the total curvature of $\Gamma$. This completes the proof when $\Gamma$ is smooth. The case of polygonal (i.e., piecewise linear) curves follows using approximation by smooth curves, and the general case then follows by taking the supremum over inscribed polygonal curves.

(Even when the curve $\Gamma$ is smooth, the annulus $A$ might not be. However, if we use a point $p^{\prime}$ in $\mathbb{R}^{N+1} \supset \mathbb{R}^{N}$, the corresponding annulus $A^{\prime}$ will be smooth. Thus the inequality will be true for $p^{\prime}$ and, letting $p^{\prime} \rightarrow p$, also for $p$. We thank M. Ghomi for this observation.)

Proof 2 (using integral geometry). We may assume $p=0$. For unit vectors $\mathbf{v}$, let $f_{\mathbf{v}}: \Gamma \rightarrow \mathbb{R}$ be the function $f_{\mathbf{v}}(x)=\mathbf{v} \cdot x$. By Crofton's formula the length of $\Pi_{p} \Gamma$ is $\pi$ times the average over all $\mathbf{v}$ of the number of zeroes of $f_{\mathbf{v}}$. Similarly, Milnor proved that the total curvature of $\Gamma$ is $\pi$ times the average over all $\mathbf{v}$ of the number of local extrema of $f_{\mathbf{v}}[\mathrm{Mi}, 3.1]$.

We claim that for each $\mathbf{v}$, the number of zeroes is $\leq$ the number of local extrema. The theorem then follows immediately by averaging over $\mathbf{v}$. To prove the claim, we may suppose $f_{\mathbf{v}}$ has finitely many extrema. To each zero $q$ of $f_{\mathbf{v}}$, associate the first local extremum $\phi(q)$ that occurs on or after $q$ in the parametrization. Note that this is an injection from the set of zeroes to the set of local extrema.

Remark 1.2. Equality in Theorem 1.1 holds if and only if (i) $\Gamma$ lies in a 2-plane through $p$, and (ii) within that 2-plane, $\Gamma$ is locally convex with respect to $p$. (Local convexity with respect to $p$ means that $\Gamma$ is a union of open arcs $U_{i}$ such that each $U_{i}$ is in the boundary of the convex hull of $U_{i}$ and $p$.)

The "if" part is easy. To prove that condition (i) is necessary for equality, suppose that $p=0$ and that $\Gamma$ is not contained in any 2-plane through 0 . This implies we can find a small arc $C$ of $\Gamma$ such that $\Pi_{p} C$ is contained in an open hemisphere of $\partial \mathbf{B}(0,1)$, but such that $\Pi_{p} C$ is not contained in the geodesic 
joining its endpoints. For such an arc, we can find a unit vector $\mathbf{v}$ such that the minimum of $f_{\mathbf{v}}$ on $C$ is positive and strictly less than its value at the two endpoints. Now (fixing $C$ ), the set of such $\mathbf{v}$ is an open set. For each such $\mathbf{v}$, note that the number of local extrema of $f_{\mathbf{v}}$ is strictly greater than the number of zeroes. (This is because the arc of $\Gamma \backslash f_{\mathbf{v}}^{-1}(0)$ that contains $C$ has not just one, but at least three local extrema; the image of the injection $\phi$ in the proof will only contain at most one of the three.) Thus when we integrate over all $\mathbf{v}$, we get strict inequality.

Necessity of (ii) is proved in a similar way.

TheOREM 1.3. Let $M$ be a minimal surface in $\mathbb{R}^{N}$ with rectifiable boundary $\Gamma$. Let $p$ be any point in $\mathbb{R}^{N}$. Then

$$
\Theta(M, p) \leq \Theta\left(\text { Cone }_{p} \Gamma, p\right)
$$

with strict inequality unless $M=\mathrm{Cone}_{p} \Gamma$.

Remark. As mentioned in the introduction, this theorem was discovered by M. Gromov [Gr, 8.2.A].

Proof. We may assume that $\Theta\left(\mathrm{Cone}_{p} \Gamma, p\right)<\infty$ or, equivalently, that

$$
\text { Length }\left(\Pi_{p} \Gamma\right)<\infty \text {. }
$$

First consider the case $p \notin \Gamma$. Let $E=E(\Gamma, p)$ denote the exterior cone over $\Gamma$ with vertex $p$ :

$$
E=\{p+t(x-p): x \in \Gamma, t \geq 1\} .
$$

Let $M^{\prime}=M \cup E$ and let

$$
\Theta\left(M^{\prime}, p, r\right)=\frac{\operatorname{area}\left(M^{\prime} \cap \mathbf{B}(p, r)\right)}{\pi r^{2}}
$$

denote the density ratio of $M^{\prime}$ in the ball $\mathbf{B}(p, r)$. Then

$$
\Theta\left(M^{\prime}, p, r\right) \leq \Theta\left(M^{\prime}, p, R\right)
$$

for $0<r<R<\infty$. For $R \leq \operatorname{dist}(p, \Gamma)$, this is the standard monotonicity theorem. For general $R$, this is the extended monotonicity theorem 9.1: $\Theta\left(M^{\prime}, p, r\right)$ is increasing, and is nonconstant unless $M^{\prime}$ is a cone with vertex $p$. Letting $r \rightarrow 0$ and $R \rightarrow \infty$ in (2) gives (1).

In case $p \in \Gamma$, the extended monotonicity formula remains true, and the proof is exactly as before, except that it is not as obvious that

$$
\lim _{r \rightarrow 0} \Theta\left(M^{\prime}, p, r\right)=\Theta(M, p) .
$$

To see that this is the case, note that

$$
\Theta\left(M^{\prime}, p, r\right)=\Theta(M, p, r)+\Theta(E, p, r) .
$$


Thus we need only show that

$$
\lim _{r \rightarrow 0} \Theta(E, p, r)=0 .
$$

This is intuitively clear if $\Gamma$ is smooth or piecewise smooth. For the general case, note that for $r \leq 1$,

$$
E \cap \mathbf{B}(p, r) \subset \operatorname{Cone}_{p}\left(\Pi_{p}(\Gamma \cap \mathbf{B}(p, r))\right),
$$

from which it follows that

$$
\Theta(E, p, r) \leq \frac{1}{2 \pi} \operatorname{Length}\left(\Pi_{p}(\Gamma \cap \mathbf{B}(p, r))\right) .
$$

Since $\Pi_{p} \Gamma$ has finite length, $A \mapsto \operatorname{Length}\left(\Pi_{p} \Gamma \mid A\right)$ defines a finite Borel measure on $G$, where $G$ is a parameter domain for $\Gamma \backslash\{p\}$. As $r \rightarrow 0, \Gamma^{-1}\left(\mathbb{R}^{N} \backslash \mathbf{B}(p, r)\right)$ exhausts $G$ and thus Length $\left(\Pi_{p}(\Gamma \cap \mathbf{B}(p, r))\right)$ tends to 0 . Therefore by (3) we get $\Theta(E, p, r) \rightarrow 0$ as required.

\section{Interior regularity}

THEOREM 2.1. Let $\Gamma$ be a simple closed curve in $\mathbb{R}^{N}$ with total curvature at most $4 \pi$. Let $M$ be a minimal surface with boundary $\Gamma$. Then the interior of $M$ is embedded, and $M$ has no interior branch points.

Proof. Since $\Gamma$ has finite total curvature, it is also rectifiable (Theorem 10.1), so we can apply Theorems 1.1 and 1.3. If $M$ is contained in a cone, then both the mean curvature and the scalar curvature vanish, so $M$ is contained in a plane. This case is trivial, so we assume $M$ is not contained in any cone, which means we have strict inequality in Theorem 1.3.

Let $p \in M \backslash \Gamma$. By Theorems 1.1 and 1.3,

$$
\Theta(M, p)<\frac{\text { TotalCurvature }(\Gamma)}{2 \pi} \leq \frac{4 \pi}{2 \pi}=2 .
$$

Thus $M$ must be unbranched and embedded at $p$, since the density of a minimal surface at any interior branch point or interior self-intersection point is at least 2 .

Corollary 2.2 (The Fáry-Milnor Theorem [Fa], [Mi]). If $\Gamma$ is a simple closed curve in $\mathbb{R}^{3}$ with total curvature at most $4 \pi$, then $\Gamma$ is unknotted.

Proof. Let $F: \mathbf{B}(0,1) \rightarrow \mathbb{R}^{3}$ be the least area disk (i.e., the Douglas-Radó solution to the Plateau problem) bounded by $\Gamma$. By the theorem, $F$ is an embedding of the interior of $\mathbf{B}(0,1)$. In particular, $r \mapsto F(\partial \mathbf{B}(0, r))$ describes an isotopy of curves for $r \neq 0$. When $r=1$, the curve is $\Gamma$, and when $r$ is near 0 , the curve is very nearly circular and is therefore unknotted. 


\section{Smooth boundaries}

In this section we exclude boundary branch points for smooth curves with total curvature at most $4 \pi$. We begin with a theorem about arbitrary curves.

TheOREM 3.1. Let $\Gamma$ be a simple closed curve in $\mathbb{R}^{N}$ with finite total curvature. Let $p$ be a point in $\Gamma$. Then

$$
\operatorname{Length}\left(\Pi_{p} \Gamma\right) \leq \text { TotalCurvature }(\Gamma)-\pi-\theta,
$$

where $\theta$ is the exterior angle to $\Gamma$ at $p$. (Thus $\theta=0, \theta=\pi$, or $0<\theta<\pi$ according to whether $\Gamma$ has a tangent line, a cusp, or a corner at $p$.)

Proof. This can be proved by a straightforward modification of the integral geometric proof of Theorem 1.1. Alternatively, we can deduce Theorem 3.1 from Theorem 1.1 as follows.

For small $r>0$, let $a=a_{r}$ and $b=b_{r}$ be the intersections of $\Gamma$ with $\partial \mathbf{B}(p, r)$. (There are only two intersection points by Theorem 10.3.) Let $\theta(r)$ be the exterior angle of the triangle $a p b$ at vertex $p$. Let $q$ be a point very close to $p$ such that $p$ is in the triangle $a q b$ but not in the segments $a q$ or $q b$.

Let $\Gamma^{\prime}=\Gamma_{r, q}^{\prime}$ be the closed curve obtained by replacing $\Gamma \cap \mathbf{B}(p, r)$ with the segments $a q$ and $q b$. By Theorem 1.1,

$$
\text { Length }\left(\Pi_{p} \Gamma_{r, q}^{\prime}\right) \leq \text { TotalCurvature }\left(\Gamma_{r, q}^{\prime}\right) \text {. }
$$

Note that $\Pi_{p} \Gamma^{\prime}$ consists of $\Pi_{p}(\Gamma \backslash \mathbf{B}(p, r))$ together with a geodesic arc of length $\pi+\theta(r)$, so

$$
\text { Length }\left(\Pi_{p}(\Gamma \backslash \mathbf{B}(p, r))\right)+\pi+\theta(r) \leq \text { TotalCurvature }\left(\Gamma_{r, q}^{\prime}\right) .
$$

Now let $q \rightarrow p$ :

$$
\begin{aligned}
\operatorname{Length}\left(\Pi_{p}(\Gamma \backslash \mathbf{B}(p, r))\right)+\pi+\theta(r) & \leq \text { TotalCurvature }\left(\Gamma_{r, p}^{\prime}\right) \\
& \leq \text { TotalCurvature }(\Gamma) .
\end{aligned}
$$

Letting $r \rightarrow 0$ gives the result.

TheOREM 3.2. Let $\Gamma$ be a smooth simple closed curve in $\mathbb{R}^{N}$ with total curvature at most $4 \pi$. Let $M$ be a minimal surface with boundary $\Gamma$. Then $M$ is a smooth embedded manifold with boundary.

Proof. We already know from Theorem 2.1 that the interior of $M$ is smooth and embedded. Let $p \in \Gamma$. As in the proof of Theorem 2.1, we may assume we have strict inequality in Theorem 1.3. Then by Theorems 1.3 and 3.1, 


$$
\Theta(M, p)<\Theta\left(\operatorname{Cone}_{p} \Gamma, p\right) \leq \frac{\text { TotalCurvature }(\Gamma)}{2 \pi}-\frac{1}{2} \leq \frac{4 \pi}{2 \pi}-\frac{1}{2}=\frac{3}{2} .
$$

But any boundary branch point has density at least $3 / 2$. Likewise, if $M$ were not embedded at $p$, then the density would be at least $3 / 2$.

\section{Boundaries with corners}

THEOREM 4.1. Let $\Gamma$ be a simple closed curve in $\mathbb{R}^{N}$ with total curvature at most $4 \pi$. Let $M$ be a minimal surface with boundary $\Gamma$.

(i) If $p$ is a point of $\Gamma$ with exterior angle $\theta$, then the density $\Theta(M, p)$ is either $\frac{1}{2}+\frac{\theta}{2 \pi}$ or $\frac{1}{2}-\frac{\theta}{2 \pi}$.

(ii) If $p$ is a cusp, then the density of $M$ at $p$ is 0 unless $\Gamma$ lies in a plane.

(iii) $M$ is embedded up to and including the boundary.

Proof. Let $T$ be a tangent cone to $M$ at $p$. Then the boundary of $T$ consists of two rays with an interior angle of $\pi-\theta$. The intersection $S$ of $T$ with the unit sphere is a collection of one or more geodesic curves (see Note 4.2 below). One curve must be a geodesic arc joining two points that are $\pi-\theta$ apart (in geodesic distance). Thus this arc must have length $\pi-\theta$ or $2 \pi-(\pi-\theta)=\pi+\theta$. The other arcs must be great circles. Thus the density of $M$ at $p$ must be

$$
\Theta(M, p)=\frac{1}{2 \pi}(\pi \pm \theta+2 \pi k)
$$

where $k \geq 0$ is the number of great circles.

Now by Theorems 1.3 and 3.1,

$$
\Theta(M, p)<\frac{1}{2 \pi}(\text { TotalCurvature }(\Gamma)-\pi-\theta) \leq \frac{1}{2 \pi}(3 \pi-\theta) .
$$

(The strict inequality comes from assuming that $M$ is not contained in a cone; otherwise all the conclusions are trivially true.) Combining (1) and (2) we see that

$$
\pi \pm \theta+2 \pi k \leq 3 \pi-\theta
$$

or

$$
(\theta \pm \theta)+2 \pi k<2 \pi .
$$

Notice this forces $k=0$. If $p$ is a cusp (so $\theta=\pi$ ), then the \pm in (3) (and therefore in (1)) must be -, which means that (in this case) the density $\Theta(M, p)$ is 0 . 
Note that if $p \in \Gamma$ coincided with an interior point of $M$, then $S$ would indeed contain at least one great circle, which we have just seen it does not. This proves the embeddedness.

Note 4.2. In the proof of Theorem 4.1 (and later in 7.1 and 7.2), we use the following well-known fact: any tangent cone to a 2-dimensional minimal variety (e.g. stationary integral varifold) intersects the unit sphere in a finite collection of geodesic arcs. This fact can be proved as follows. The intersection of the tangent cone with the unit sphere is a 1-dimensional varifold that is, by a straightforward calculation, stationary in the sphere. By [AA], such 1dimensional stationary varifolds consist of geodesic arcs.

\section{Nondisk type surfaces}

In this section we prove that there are simple closed curves in $\mathbb{R}^{3}$ with total curvature $<4 \pi$ that bound minimal Möbius strips.

Consider two convex polygons in the halfplane $\{(x, y, 0): y \geq 0\}$ such that each polygon has exactly one vertex, namely the origin, located on the $x$-axis. (Think of the two polygons being the same regular $n$-gon.) Give both polygons the counterclockwise orientation. Now rotate one of the two polygons about the $x$-axis through a small positive angle and the other through a small negative angle. The two polygons will still have the origin as a common vertex.

Consider the closed connected curve $\Gamma$ that, starting at the origin, traces out one polygon and then the other. We claim that $\Gamma$ has total curvature $<4 \pi$. To see why, note that for a generic unit vector $\mathbf{v}$, the function $f_{\mathbf{v}}(x)=\mathbf{v} \cdot x$ will not be constant on any segment of $\Gamma$. For such a $\mathbf{v}, f_{\mathbf{v}}$ can have at most four local extrema (two on each polygon). However, the set of such $\mathbf{v}$ for which $f_{\mathbf{v}}$ has only two local extrema is open and nonempty since it contains vectors arbitrarily close to $(0,0,1)$. Thus the average number of local extrema is less than 4, which implies [Mi, 3.1] that the total curvature is less than $4 \pi$.

Of course $\Gamma$ has a self-intersection, but we can move one vertex slightly to make it embedded. We can also round the corners to make it smooth or, by further perturbation, even analytic.

Note we can construct such a $\Gamma$ that, after a suitable translation, is arbitrarily close to a curve that traverses the unit circle in the $x y$ plane twice. We can also make $\Gamma$ lie outside the unit cylinder $\mathbf{B}(0,1) \times \mathbb{R}$. Fix such a curve $\Gamma$.

Now a disk (or any other orientable surface) bounded by $\Gamma$ has area $\geq 2 \pi$, since its projection to the $x y$-plane must cover the unit disk twice algebraically. But clearly $\Gamma$ bounds a Möbius strip with much smaller area. Thus by the unoriented version of the Douglas theorem (see [Sh1] or [Be]), $\Gamma$ bounds a minimal Möbius strip $M$. 
If we perturb $\Gamma$ slightly, then it will not bound any minimal surfaces with nonzero nullity (by Theorem 11.1(i)). Hence $M$ will be strictly stable. It then follows from degree theory $(\S 11.1($ ii) $)$ that $\Gamma$ must also bound an unstable minimal Möbius strip.

By analogy with certain results and conjectures [MW] about minimal surfaces bounded by pairs of convex curves, we make the following conjecture.

CONJECTURE. Let $\Gamma$ be a smooth simple closed curve in $\mathbb{R}^{N}$ with total curvature $\leq 4 \pi$. Then in addition to a unique minimal disk, $\Gamma$ bounds either:

(i) no other minimal surfaces, or

(ii) exactly one minimal Möbius strip and no other minimal surfaces, or

(iii) exactly two minimal Möbius strips and no other minimal surfaces.

In case (ii), the strip has index 0 and nullity 1 . In case (iii), both strips have nullity 0 , one has index 1 and the other has index 0.

We emphasize that this conjecture refers to classical minimal surfaces bounded by $\Gamma$ : in Section 7 we show that $\Gamma$ can also bound soap-film-like minimal varieties.

It would be interesting to know the least possible total boundary curvature of a minimal Möbius strip, or even a good lower bound. One could ask the same question about higher genus nonorientable surfaces, although, if the above conjecture is correct, the answer would be $4 \pi$ by the Almgren-ThurstonHubbard examples.

\section{Disconnected boundaries}

Suppose $M$ is a minimal surface with more than one boundary component, and suppose that the total curvature of the boundary (i.e., the sum of the total curvatures of the components) is at most $4 \pi$. By the Fenchel-Borsuk theorem ([Fen], [Bor], [Mi], [Fa]), each component has total curvature $\geq 2 \pi$ and indeed has total curvature $>2 \pi$ unless it is a plane convex curve. Thus $\partial M$ must consist of exactly two components $\Gamma_{1}$ and $\Gamma_{2}$, each of which is a plane convex curve.

If $M$ is a cone, then its scalar curvature and mean curvature both vanish, so it is locally planar. That is, it is the union of the two planar regions $R_{1}$ and $R_{2}$ bounded by $\Gamma_{1}$ and $\Gamma_{2}$. In this case, the two regions must intersect since the vertex of the cone belongs to both regions. If $M$ is not a cone, then all the conclusions of Theorems 2.1, 3.2, and 4.1 hold, with exactly the same proofs. (Connectivity of $\partial M$ was only used to conclude that $M$ was not a cone.) 
Meeks and White $[\mathrm{MW}]$ studied the case when $\Gamma \subset \mathbb{R}^{3}$ and $R_{1}$ and $R_{2}$ are disjoint. They proved, for example, that such a $\Gamma$ bounds at most two minimal surfaces of annular type, and that only one of the annuli can be stable. They also conjectured that such a $\Gamma$ cannot bound minimal surfaces of higher genus. If, on the other hand, $R_{1}$ and $R_{2}$ are not disjoint but rather intersect along a segment, then $\Gamma$ will bound other surfaces. In particular, there are examples [MW, 3] that bound more than one stable annulus. Futhermore, by the heuristic principle that every minimal surface with a curve of selfintersection should be the limit (as a set) of a sequence of embedded minimal surfaces, we expect that such a $\Gamma$ should bound embedded minimal surfaces of arbitrarily large genus. (Apply the heuristic principle to $R_{1} \cup R_{2}$.)

\section{Nonclassical minimal surfaces}

As we have seen, a smooth simple closed curve of finite total curvature $\leq 4 \pi$ does not bound any classical minimal surface with branch points or with self-intersections. However, it may bound other minimal varieties, such as stationary integral varifolds and flat chains modulo $k$, that do have singularities.

For example, consider a curve $\Gamma_{0}$ in $\mathbb{R}^{3}$ that traverses the unit circle in the $x y$ plane twice and therefore has total curvature $4 \pi$. As shown in Section 5 , one can modify the curve slightly to get an embedded curve $\Gamma$ with total curvature less than $4 \pi$ that lies just outside the cylinder $\mathbf{B}(0,1) \times \mathbb{R}$.

Let $T$ be a mod 3 area minimizing surface (i.e., flat chain) with boundary $\Gamma$. If $T$ were smooth, it would be orientable ( since $1 \not \equiv-1 \bmod 3)$, and so its area would be $\geq 2 \pi$ since its projection to the $x y$ plane would have to cover the unit disk twice algebraically.

However, $T$ has area at most slightly greater than $\pi$. To see this, consider the surface $T^{\prime}$ consisting of a ribbon homotoping $\Gamma$ to $\Gamma_{0}$, together with the disk $\mathbf{B}(0,1)$ with multiplicity -1 . Then the boundary of $T^{\prime}$ is congruent to $\Gamma$ $\bmod 3$, and the mass is only slightly more than $\pi$. Thus $T$ cannot be smooth.

Now consider a rectifiable curve $\Gamma$ in $\mathbb{R}^{N}$. Recall that a compactly supported rectifiable varifold $M$ is said to be stationary in $\mathbb{R}^{N} \backslash \Gamma$ if

$$
\left(\frac{d}{d t}\right)_{t=0} \operatorname{area}(\phi(t, M))=0
$$

for all smooth $\phi: \mathbb{R} \times \mathbb{R}^{N} \rightarrow \mathbb{R}^{N}$ such that $\phi(0, x) \equiv x$ and $\phi(t, \xi) \equiv \xi$ for $\xi$ in a neighborhood of $\Gamma$. Let us call $M$ strongly stationary with respect to $\Gamma$ provided

$$
\left(\frac{d}{d t}\right)_{t=0}(\operatorname{area}(\phi(t, M))+\operatorname{area}(\phi([0, t] \times \Gamma))) \geq 0
$$


whenever $\phi: \mathbb{R} \times \mathbb{R}^{N} \rightarrow \mathbb{R}^{N}$ is a smooth map with $\phi(0, x) \equiv x$. Intuitively, strong stationarity means that the area of $M$ cannot be reduced to first order by any smooth deformation, even moving the boundary, provided we include the ribbon swept out by $\Gamma$ as part of the deformed surface.

Strong stationarity may also be stated in any of the following three equivalent ways:

(i) For any smooth map $\phi: \mathbb{R} \times \mathbb{R}^{N} \rightarrow \mathbb{R}^{N}$ with $\phi(0, x) \equiv x$, we have

$$
\left(\frac{d}{d t}\right)_{t=0} \operatorname{area}(\phi(t, M)) \leq \int_{\Gamma}\left|X^{\perp}\right| d s,
$$

where $X(x)=\left(\frac{d}{d t}\right)_{t=0} \phi(t, x)$ and $X^{\perp}$ is the component of $X$ normal to $\Gamma$. (ii) $\int_{M} \operatorname{div}_{M} X \leq \int_{\Gamma}\left|X^{\perp}\right| d s$ for smooth vectorfields $X$ on $\mathbb{R}^{N}$.

(iii) There is an $\mathcal{H}^{1}$-measurable normal vectorfield $\nu$ on $\Gamma$ with sup $|\nu| \leq 1$ such that $\int_{M} \operatorname{div}_{M} X=\int_{\Gamma} X \cdot \nu d s$ for all smooth vectorfields $X$ on $\mathbb{R}^{N}$.

The integrals over $\Gamma$ are with respect to arclength or, equivalently, one-dimensional Hausdorff measure. The equivalence of strong stationarity and (i) is clear. Statements (i) and (ii) are equivalent by the first variation formula, and (ii) and (iii) are equivalent by the Riesz representation theorem.

We emphasize (as evidenced by (ii) and (iii)) that strong stationarity is really stationarity in $\mathbb{R}^{N} \backslash \Gamma$ plus a boundary condition. Indeed, for $C^{1,1}$ curves $\Gamma$, the boundary condition is that the first variation boundary measure is $\leq$ arclength measure on $\Gamma$. (This would be true for general rectifiable $\Gamma$ if the varifold-theoretic unit normal at the boundary were known to be perpendicular to the boundary almost everywhere. For the $C^{1,1}$ case, see [All2].)

Thinking physically, one could say that $M$ is strongly stationary if it is stationary, if the total force per unit length that the surface exerts on $\Gamma$ is everywhere $\leq 1$, and if this force is perpendicular to the boundary.

To better understand this definition, note that any classical minimal surface with smooth boundary $\Gamma$ is (as a varifold) strongly stationary with respect to $\Gamma$. The union of two such surfaces with the same boundary will not in general define a strongly stationary varifold. Indeed, the resulting varifold is strongly stationary if and only if the angle between the the two surfaces is everywhere $\geq 120$ degrees along the boundary. (To see this, use the first variation formula.) This corresponds to what one observes in soap films: two sheets can meet along a portion of wire, but never with an angle of less than 120 degrees.

THEOREM 7.1. Let $\Gamma$ be a simple closed curve in $\mathbb{R}^{N}$ with total curvature at most $3 \pi$. Suppose $M$ is a compactly supported rectifiable varifold that is strongly stationary with respect to $\Gamma$. Suppose also that the density of $M$ is $\geq 1$ at every point in (support $M) \backslash \Gamma$. Then $M$ is smooth in the interior, and also at the boundary if the boundary is smooth. 
Proof. As in the proof of Theorem 2.1, we can assume that $M$ is not contained in a cone. Consider first a point $p \notin \Gamma$. Then

$$
\Theta(M, p)<\Theta\left(\text { Cone }_{p} \Gamma, p\right) \leq \frac{1}{2 \pi} \text { TotalCurvature }(\Gamma) \leq \frac{3}{2} .
$$

(The first inequality is the varifold version of Theorem 1.3: the proof is identical, except that one uses the varifold version $(\S 9.2)$ of the extended monotonicity formula.) But this density bound implies that $p$ is regular as follows:

Let $C$ be a tangent cone at $p$. Then $\Theta(C, 0)<3 / 2$. Thus $\Theta(C, x)<3 / 2$ for all $x$, since in any minimal cone, the highest density occurs at the vertex. (This is because the density function $\Theta(C, \cdot)$ is upper-semicontinuous [Si, $\S 17.8]$ and constant along radial lines.) Now the intersection of $C$ with the unit sphere is a collection of geodesic arcs $(\S 4.2)$, which means that the cone $C$ is polyhedral. At most two faces of $C$ can meet along an edge, since otherwise the density at points in the edge would be $\geq 3 / 2$. This means $C \cap \partial \mathbf{B}$ is a union of great circles. Since the density is $<3 / 2$, there is only one great circle and it has multiplicity 1. By Allard's regularity theorem ([All1] or [Si, §24.2]), this means that $M$ is regular at $p$.

The boundary case is similar: one shows that the density at any boundary point is $<1$, whereas the minimum possible density at a boundary singularity is 1 .

This theorem becomes false if $3 \pi$ is replaced by $4 \pi$ : the varifold associated to the mod 3 flat chain $T$ discussed at the beginning of this section is a counterexample.

The surface $M$ in Theorem 7.1 is not assumed to minimize area in any sense. For surfaces in $\mathbb{R}^{3}$ that minimize area modulo $k$, less control on total boundary curvature is needed to ensure regularity:

THEOREM 7.2. Let $\Gamma$ be an embedded closed curve (not necessarily connected) in $\mathbb{R}^{3}$ with total curvature $\leq k \pi$. Let $M$ be a mass-minimizing surface $\bmod k$ with boundary $\Gamma$. Then $M$ is smooth and embedded in the interior, and also at the boundary if the boundary is smooth.

Proof. Consider a tangent cone $C$ to $M$ at a point $p \notin \Gamma$. As in the proof of Theorem 7.1, $C$ must be a polyhedral complex. If the complex has edges, then $k$ (or a multiple of $k$ ) sheets meet along such an edge which (as in the beginning of this section for the case $k=3$ ) means that $C$ has density $\geq k / 2$. Thus if $C$ has density $<k / 2$, it has no edges and is therefore a plane with some multiplicity $<k / 2$. 
Now, as in the proof of Theorem 7.1, one deduces that the density at each interior point $p$ of $M$ is $<k / 2$. Let $C$ be a tangent cone to $M$ at $p$. Then, as we have just seen, $C$ is a plane of multiplicty $<k / 2$. But that implies that $p$ is a regular point of $M$ [Wh1, Theorem 3.1].

Thus the interior of $M$ is a smooth minimal surface. Since the density is everywhere $<k / 2, M$ is orientable. Consequently $M$ is mass-minimizing as an integral current. Thus it is smooth at the boundary wherever the boundary is smooth [HS].

Does this theorem also hold in $\mathbb{R}^{N}$ for $N>3$ ? The proof of the main theorem of [Wh1] is valid only for hypersurfaces. However, that theorem is probably true for arbitrary codimensions. If so, then Theorem 7.2 does indeed hold for surfaces in $\mathbb{R}^{N}$.

Probably neither of these last two theorems (7.1 or 7.2) is sharp. However, as $k \rightarrow \infty$ the ratio of the bound in Theorem 7.2 to the optimal bound tends to 1 . For consider an embedded curve that is a slight perturbation of a circle traversed $(k+1) / 2$ times if $k$ is odd or $(k+2) / 2$ times if $k$ is even. Then the total curvature is slightly less than $(k+1) \pi$ or $(k+2) \pi$, respectively, and the mass minimizing surface mod $k$ will have singularities. The proof is just like the proof for the special case $k=3$ given at the beginning of this section.

Morgan [Mo1] proved an interesting theorem related to Theorem 7.2: if an $m$-dimensional mass minimizing hypersurface $\bmod p$ has a smooth, extreme boundary with at most $p / 2$ connected components, then the hypersurface is smooth except for a set of Hausdorff dimension $\leq m-7$.

\section{Nonclosed curves}

THEOREM 8.1. Let $\Gamma$ be a smooth embedded connected nonclosed curve in $\mathbb{R}^{N}$ with total curvature $\leq 2 \pi$. Then no nontrivial varifold $M$ with bounded support and with density $\geq 1$ throughout (support $M) \backslash \Gamma$ is strongly stationary with respect to $\Gamma$.

This is sharp because for every $\varepsilon>0$, there are examples (see [DW]) with total curvature $\leq 2 \pi+\varepsilon$. Readers can construct such examples experimentally by tying a simple overhand knot in a piece of wire and dipping it in soapy water.

Proof. Suppose $M$ were such a varifold. We may assume that $\Gamma$ is compact; otherwise replace $\Gamma$ by the smallest connected subset of $\Gamma$ that contains $\Gamma \cap \operatorname{support} M$. We claim that

$$
\Theta\left(\text { Cone }_{p} \Gamma, p\right) \leq \frac{\text { TotalCurvature }(\Gamma)+\pi}{2 \pi}
$$


for any nonclosed curve $\Gamma$ and point $p \notin \Gamma$. Using this (and the analogous inequality at the boundary), one shows exactly as in the proof of Theorem 7.1 that $M$ is a smooth embedded manifold with boundary. But then the boundary must be a closed curve. Since $\Gamma$ contains no closed curve, this means that the boundary of $M$ is empty. But there are no compact, boundaryless minimal surfaces in $\mathbb{R}^{N}$, a contradiction.

To see $(*)$, attach long line segments to the ends of $\Gamma$ to get a new curve with endpoints in a large sphere $\mathbf{B}(p, R)$ centered at $p$ and with the same total curvature. Now join the ends by a geodesic arc in $\mathbf{B}(p, R)$ to get a closed curve $\Gamma^{\prime}$. The total curvature of $\Gamma^{\prime}$ will exceed that of $\Gamma$ by approximately $\pi$ (for the two new corners) plus the angle $\alpha$ subtended by the geodesic arc. The length of $\Pi_{p} \Gamma^{\prime}$ will exceed that of $\Pi_{p} \Gamma$ by at least $\alpha$. Hence applying Theorem 1.1 (or Theorem 3.1 if $p \in \Gamma^{\prime}$ ) to $\Gamma^{\prime}$ and then letting $R \rightarrow \infty$ gives $(*)$.

Drachman and White [DW] proved (when $N=3$ ) the stronger result that there is no compactly supported rectifiable varifold that is stationary in $\mathbb{R}^{N} \backslash \Gamma$.

\section{The extended monotonicity theorem}

TheOREM 9.1. Let $M$ be a minimal surface in $\mathbb{R}^{N}$ such that $\Gamma=\partial M$ has finite length, and let $p$ be a point in $\mathbb{R}^{N}$. Let $M^{\prime}$ be the union of $M$ and the exterior cone $E$ over $\Gamma$ with vertex $p$. Then

$$
\Theta\left(M^{\prime}, p, r\right):=\frac{\operatorname{area}\left(M^{\prime} \cap \mathbf{B}(p, r)\right)}{\pi r^{2}}
$$

is an increasing function of $r$, and is not constant unless $M^{\prime}$ is a cone.

Note. If $\gamma: S^{1} \rightarrow \mathbb{R}^{N}$ is a Lipschitz parametrization of $\partial M$ (e.g., parametrization by arclength), then $E$ is given by the mapping

$$
\begin{aligned}
& e: S^{1} \times[1, \infty) \rightarrow \mathbb{R}^{N}, \\
& e(s, \lambda)=\lambda \gamma(s) .
\end{aligned}
$$

Of course the area referred to in $(*)$ is mapping area (or, equivalently, area of the image counting multipicities).

In the case of area-minimizing surfaces $M$, the extended monotonicity theorem appeared in [Wh4].

Proof. The proof of the monotonicity formula in [Si, §17] for manifolds without boundary also works for manifolds with boundary, provided a boundary term is added. More precisely, suppose $\Sigma \subset \mathbb{R}^{N}$ is a smooth submanifold with boundary. Let $H$ be its mean curvature vector, $\mathbf{n}_{\Sigma}(x)$ be the outward 
pointing unit normal to $\partial \Sigma$ at $x$ (that is, $\mathbf{n}_{\Sigma}(x)$ is tangent to $\Sigma$, normal to $\partial \Sigma$, and points out of $\Sigma$ ), and let $\rho(x)=|x-p|$. Then

$$
\begin{aligned}
\frac{d}{d r}(\Theta(\Sigma, p, r))= & \frac{d}{d r} \int_{\Sigma \cap \mathbf{B}(p, r)} \frac{\left|D^{\perp} \rho\right|^{2}}{\rho^{2}} d A \\
& +r^{-3} \int_{\Sigma \cap \mathbf{B}(p, r)}(x-p) \cdot H d A-r^{-3} \int_{\partial \Sigma \cap \mathbf{B}(p, r)}(x-p) \cdot \mathbf{n}_{\Sigma} d s .
\end{aligned}
$$

On the other hand, straightforward calculation shows that if $\Sigma$ is the exterior cone with vertex $p$ over a piecewise smooth curve $\partial \Sigma$, then

$$
\frac{d}{d r}(\Theta(\Sigma, p, r))=-r^{-3} \int_{\partial \Sigma \cap \mathbf{B}(p, r)}(x-p) \cdot \mathbf{n}_{\Sigma} d s .
$$

Now, consider first the case that $\Gamma$ is smooth or piecewise smooth. Then we can apply formulas (1) and (2) to $M$ and $E$, respectively. Note the term involving $H$ vanishes for $M$ since it is minimal. Thus adding the formulas gives

$$
\begin{aligned}
\frac{d}{d r}\left(\Theta\left(M^{\prime}, p, r\right)\right)= & \frac{d}{d r} \int_{M \cap \mathbf{B}(p, r)} \frac{\left|D^{\perp} \rho\right|^{2}}{\rho^{2}} d A \\
& -r^{-3} \int_{\Gamma \cap \mathbf{B}(p, r)}(x-p) \cdot\left(\mathbf{n}_{M}+\mathbf{n}_{E}\right) d s .
\end{aligned}
$$

Let $T(x)$ be a unit tangent vector to $\partial M$ at $x$. Consider the quantity

$$
(x-p) \cdot \mathbf{n}
$$

as a funtion of unit vectors $\mathbf{n}$ perpendicular to $T(x)$. Note that the minimum and maximum occur at $\mathbf{n}=\mathbf{n}_{E}$ and $\mathbf{n}=-\mathbf{n}_{E}$, respectively. Hence

$$
(x-p) \cdot\left(\mathbf{n}_{M}+\mathbf{n}_{E}\right) \leq(x-p) \cdot\left(-\mathbf{n}_{E}+\mathbf{n}_{E}\right)=0
$$

thus (3) becomes:

$$
\frac{d}{d r}\left(\Theta\left(M^{\prime}, p, r\right)\right) \geq \frac{d}{d r} \int_{M \cap \mathbf{B}(p, r)} \frac{\left|D^{\perp} \rho\right|^{2}}{\rho^{2}} d A
$$

or

$$
\Theta\left(M^{\prime}, p, R\right)-\Theta\left(M^{\prime}, p, r\right) \geq \int_{M \cap(\mathbf{B}(p, R) \backslash \mathbf{B}(p, r))} \frac{\left|D^{\perp} \rho\right|^{2}}{\rho^{2}} d A .
$$

This proves the result if $\partial M$ is smooth or piecewise smooth.

Now suppose that $\partial M$ is merely rectifiable. Then (see the note below) we can exhaust $M$ by subdomains $M_{i}$ such that $\partial M_{i}$ is smooth, $\partial M_{i}$ converges to $\partial M$ uniformly, and the length of $\partial M_{i}$ converges to the length of $\partial M$. 
It follows that $M_{i}$ and $E_{i}$ converge to $M$ and $E$ as measures and therefore that $\Theta\left(M_{i}^{\prime}, p, r\right)$ converges to $\Theta\left(M^{\prime}, p, r\right)$ for almost every $r$. Fix $r$ and $R$ with $r<R$ for which the convergence holds. By (4) applied to $M_{i}^{\prime}$,

$$
\begin{aligned}
\Theta\left(M_{i}^{\prime}, p, R\right)-\Theta\left(M_{i}^{\prime}, p, r\right) & \geq \int_{M_{i} \cap(\mathbf{B}(p, R) \backslash \mathbf{B}(p, r))} \frac{\left|D^{\perp} \rho\right|^{2}}{\rho^{2}} d A \\
& \geq \int_{M_{j} \cap(\mathbf{B}(p, R) \backslash \mathbf{B}(p, r))} \frac{\left|D^{\perp} \rho\right|^{2}}{\rho^{2}} d A
\end{aligned}
$$

for $j<i$ since then $M_{j} \subset M_{i}$. Letting first $i$ and then $j$ tend to infinity gives (4) for $M^{\prime}$ itself.

Note. If $M$ is a disk, then the fact that $\partial M$ can be approximated in $M$ by a smooth curve of nearly the same arclength is due to Radó [Ra1]. (Radó's proof is also given in $[\mathrm{N} 4, \S 316]$.) For general $M$, we indicate a quick proof similar to Radó's. Let $R$ be an annular region in $M$, one component of which is a connected component of $\partial M$ and the other a smooth curve. Then $R$ can be parametrized conformally by a map

$$
F:(x, y) \in A=S^{1} \times[0, a] \rightarrow \mathbb{R}^{N} .
$$

Of course since $M$ is minimal, $F$ will also be harmonic. We claim that the arclength $L(y)$ of $F(\cdot, y)$ is a convex function of $y$. Now $\frac{\partial F}{\partial x}$ is harmonic, so $\left|\frac{\partial F}{\partial x}\right|$ is subharmonic. (Of course at the boundary of $A$, this has to be understood as a distribution or measure.) Note that $(x, y) \mapsto L(y)$ is the average of the subharmonic functions $\left|\frac{\partial F}{\partial x}(x+\theta, y)\right|$ (averaged over $\theta$ ) and hence is subharmonic. But $L(y)$ depends only on $y$, so subharmonicity is equivalent to convexity.

Distribution derivatives at the boundary may be avoided by replacing $\left|\frac{\partial F}{\partial x}\right|$ by the subharmonic function $\sum_{j=0}^{n}\left|F\left(w_{n}^{j+1} x, y\right)-F\left(w_{n}^{j} x, y\right)\right|$ where $w_{n}=$ $\exp (2 \pi i / n)$, averaging as above, and then letting $n \rightarrow \infty$.

Convexity of the function $L(y)$ was first proved, using a somewhat more complicated argument, by Osserman and Schiffer [OS]. They also proved the sharper result that $L^{\prime \prime}(y) \geq L(y)$, with equality precisely for catenoids.

9.2. Varifolds. The extended monotonicity theorem is also true for $m$ dimensional rectifiable varifolds $V$ that are strongly stationary (see $\S 7$ ) with respect to a closed rectifiable set $\Gamma$. Of course if $m \neq 2$, then the expression $\pi r^{2}$ in $9.1(*)$ should be replaced by $\omega_{m} r^{m}$ where $\omega_{m}$ is the volume of the unit ball in $\mathbb{R}^{m}$. The proof is exactly as above. The exterior cone should be interpreted as the varifold image of the multiplicity 1 varifold associated with $\Gamma \times[1, \infty)$ under the map $e$ defined at the beginning of this section. 
9.3. Mass minimizing flat chains. The extended monotonicity theorem is also true for $m$-dimensional mass minimizing integral currents or, more generally, for mass minimizing flat chains with coefficients in any normed group. The exterior cone should be interpreted as the flat chain image $e_{\#}(\partial M \times[1, \infty))$, where $e$ is as in the note at the beginning of this section. As in $\S 9.2$, if $m \neq 2$, then the expression $\pi r^{2}$ in $9.1(*)$ should be replaced by $\omega_{m} r^{m}$, where $\omega_{m}$ is the volume of the unit ball in $\mathbb{R}^{m}$. The proof is exactly like the proof by cone comparison (cf. $[\mathrm{Mo} 2,9.3]$ ) of the usual monotonicity formula.

\section{Two basic properties of curves with finite total curvature}

\section{Rectifiability.}

THEOREM 10.1. If $\Gamma$ is a compact connected curve in $\mathbb{R}^{N}$ with finite total curvature, then it has finite length.

Proof. Let $\gamma(t)$ be a parametrization of $\Gamma$. We may assume that $\gamma$ is closed (otherwise close it up with a straight line segment). If $\mathbf{u}$ is a unit vector, then the total variation of $t \mapsto \gamma(t) \cdot \mathbf{u}$ is at most the diameter of $\Gamma$ times the number of local extrema of $\gamma(t) \cdot \mathbf{u}$. Averaging both sides of this inequality (over all unit vectors $\mathbf{u})$ gives

$$
c_{N} \operatorname{Length}(\Gamma) \leq(\operatorname{diam} \Gamma) \cdot \operatorname{TotalCurvature}(\Gamma),
$$

where $c_{N}>0$ depends only on the dimension $N$. (Here, as in the proof of Theorem 1.1, we are using the integral geometric formulas of Crofton and Milnor [Mi, 3.1].)

\section{Existence of strong one-sided tangents.}

Lemma 10.2. Suppose $\gamma:[a, b] \rightarrow \mathbb{R}^{N}$ is an injectively parametrized curve of finite total curvature. For $\xi<\eta$ in $[a, b]$, let

$$
T_{\xi \eta}=\frac{\gamma(\eta)-\gamma(\xi)}{|\gamma(\eta)-\gamma(\xi)|}
$$

If $a<x \leq y<b$, then the angle $\angle\left(T_{a x}, T_{y b}\right)$ between $T_{a x}$ and $T_{y b}$ is less than or equal to the total curvature of $\gamma \mid(a, b)$.

Proof.

$$
\begin{aligned}
\operatorname{TotalCurvature}(\gamma \mid(a, b)) & \geq \angle\left(T_{a x}, T_{x y}\right)+\angle\left(T_{x y}, T_{y b}\right) \\
& \geq \angle\left(T_{a x}, T_{y b}\right)
\end{aligned}
$$

by the triangle inequality for geodesic distances in the unit sphere. 
Theorem 10.3. Suppose $\gamma:[A, B] \rightarrow \mathbb{R}^{N}$ is an injectively parametrized curve of finite total curvature. Then the strong one-sided tangents

$$
T^{+}(a)=\lim _{\substack{a \leq x<y \\ y \rightarrow a}} T_{x y} \quad \text { and } \quad T_{-}(b)=\lim _{\substack{x<y \leq b \\ x \rightarrow b}} T_{x y}
$$

exist for every $a \in[A, B)$ and $b \in(A, B]$. Furthermore, $T^{+}(x)$ and $T_{-}(x)$ both approach $T^{+}(a)$ as $x$ approaches a from the right (i.e, with $\left.x>a\right)$, and they both approach $T_{-}(b)$ as $x$ approaches $b$ from the left (i.e., with $x<b$ ).

Remark. After completing this paper, we discovered that this lemma appears as lemma 2.13 in $[\mathrm{Ku}]$. We include the proof below to keep the paper self-contained and because it seems to us more direct than the proof in $[\mathrm{Ku}]$.

Proof. Let $T$ be a subsequential limit of $T_{a x}$ as $x \rightarrow a$ with $x>a$. Thus by lemma 10.2 applied to $\gamma \mid[a, y]$,

$$
\angle\left(T, T_{x y}\right) \leq \text { TotalCurvature }(\gamma \mid(a, y))
$$

for $a<x<y<b$. Notice that as $y \rightarrow a$ with $y>a$, the right-hand side goes to 0 . This proves that $T^{+}(a)=T$ exists. Likewise $T_{-}$exists at every point.

Letting $x \rightarrow y$ and then $y \rightarrow a$ in the definition (1) of $T^{+}(a)$, we immediately read off that $T_{-}(y) \rightarrow T^{+}(a)$ as $y \rightarrow a$. If, however, we let $y \rightarrow x$ and then $x \rightarrow a$, we get that $T^{+}(x) \rightarrow T^{+}(a)$. The convergence to $T_{-}(b)$ from the left is proved analogously.

Remark 10.4. It follows from (2) that if $a \leq x<y \leq b$, then the angle between $T_{x y}$ and $T_{a b}$ is at most $2 \kappa(a, b)$, where $\kappa(a, b)$ is the total curvature of $\gamma \mid(a, b)$. Consequently, the length of $\gamma(a, b)$ is at most $1 / \cos (2 \kappa(a, b))$ times $|\gamma(b)-\gamma(a)|$ provided $\kappa(a, b)<\pi / 4$. (This incidentally can be used to give another proof of Theorem 10.1.) It follows that $T^{+}(a)$ is the right derivative of $\gamma$ with respect to arclength. Likewise $T_{-}(a)$ is the corresponding left derivative.

\section{The collection of minimal Möbius strips spanning a given curve}

TheOREM 11.1. Let $\mathcal{G}$ be the set of smooth simple closed curves in $\mathbb{R}^{N}$ with total curvature $<4 \pi$. Let $\mathcal{N}$ be the set of curves $\Gamma$ in $\mathcal{G}$ that bound minimal Möbius strips with nonzero nullity. Then:

(i) the set $\mathcal{N}$ is closed and nowhere dense, and

(ii) each curve $\Gamma \in \mathcal{G} \backslash \mathcal{N}$ bounds only finitely many minimal Möbius strips, exactly half of which have even index. 
Proof. Let $\mathcal{M}$ be the set of all minimal Möbius strips bounded by curves in $\mathcal{G}$, and let $\Pi$ be the forgetful map from $\mathcal{M}$ to $\mathcal{G}$ :

$$
\begin{aligned}
& \Pi: \mathcal{M} \rightarrow \mathcal{G}, \\
& \Pi: M \mapsto \partial M .
\end{aligned}
$$

By Theorem 3.2, the elements of $\mathcal{M}$ are all smooth and embedded. Thus by [Wh2, $\S 1.5$ and $\S 3.3], \mathcal{M}$ has the structure of a separable Banach manifold, and $\Pi$ is a smooth Fredholm map of Fredholm index 0.

We claim that the map $\Pi$ is proper. To see this, let $M_{i}$ be a sequence of surfaces in $\mathcal{M}$ with $\Pi\left(M_{i}\right)=\partial M_{i}$ converging smoothly to $\Gamma \in \mathcal{G}$. By the Gauss-Bonnet theorem, the surfaces in $\mathcal{M}$ have uniformly bounded total curvature. Hence a subsequence of the $M_{i}$ will converge smoothly to a limit surface $M$ except at isolated singular points of $M$. (See parts (1) and (2) of Theorem 3 in [Wh3]. The theorem is stated only for 3-manifolds, but the proof of parts (1) and (2) is valid for minimal surfaces in $N$-manifolds.) Since $M$ has finite topological type, the singularities can only be branch points. (See [O2, Theorem 1] for interior singularities; a slight modification of the proof there establishes the result for boundary singularities.) But by Theorem 3.2, $M$ has no branch points. Hence $\Pi$ is a proper map.

By the Sard-Smale theorem [Sm] or by [Wh2, §3.3(6)], the set of critical values of $\Pi$ is a countable union of closed nowhere dense sets. By the properness of $\Pi$, in fact this set must be closed and nowhere dense. By [Wh2, §3.3], the set of critical values is precisely $\mathcal{N}$. This proves (i).

We also claim that the space $\mathcal{G}$ is path connected. The properness of $\Pi$ then implies [Wh2, $\S 3.3$ ] that $\Pi$ has a mapping degree $d$ that, for any noncritical $\Gamma$, is equal to the difference between the numbers of even and odd index minimal Möbius strips bounded by $\Gamma$. If $\Gamma$ is a planar curve, then it bounds no minimal Möbius strips. Thus $d$ must be 0 . This proves (ii), given the path connectivity of $\mathcal{G}$.

Thus it remains only to show that $\mathcal{G}$ is path connected. That is, we must show that a curve $\Gamma$ in $\mathcal{G}$ can be deformed through curves in $\mathcal{G}$ to a plane convex curve (for example). By Milnor, there is a linear function $L$ on $\mathbb{R}^{N}$ such that the restriction of $L$ to $\Gamma$ has exactly one local maximum and one local minimum. We may also assume that the maximum is nondegenerate. Let $[a, b]$ be the image of $\Gamma$ under $L$. For $t \in[a, b)$, let $\Gamma(t)$ be the curve obtained by replacing the portion of $\Gamma$ where $L \leq t$ by a straight line segment. Note that each $\Gamma(t)$ is embedded. Also, this truncation can only decrease total curvature, so $\Gamma(t)$ has total curvature less than $4 \pi$. When $t=a, \Gamma(t)$ is the original curve, and when $t$ is close to $b, \Gamma(t)$ is very nearly a convex plane curve (namely, half of an ellipse). Of course $\Gamma(t)$ is only piecewise smooth. We leave it to the reader to modify the deformation so that each curve is smooth and so that the final curve is planar and convex. 


\section{Remarks}

11.2. The theorem also holds, with the same proof, for surfaces of any other topological type except the disk type. However, if the conjecture in Section 5 is correct, then there are no such surfaces.

11.3. The theorem also holds, with the same proof, for disk-type surfaces, except that in conclusion (ii) of the theorem, the number of even index disks will be one more than the number of odd index disks. Of course when $N=3$, there is only one disk by Nitsche's theorem.

11.4. For orientable surfaces, these assertions (11.2 and 11.3) follow immediately from work of Tomi and Tromba ([TT3]). Moreover, for $N \geq 4$, the assumption on total curvature is not needed. Presumably the theory in [TT3] could be modified for nonorientable surfaces.

11.5. Theorem 11.1 could presumably be proved by minimax theorems as in [Sh2] or [PR]. However, [Sh2] assumes orientability, and details of [PR] have not yet appeared.

UpPSAla University, 75206 UpPSAla, SwEDEN

E-mail address: tobias@math.uu.se

Stanford University, Stanford, CA, USA

E-mail address: white@math.stanford.edu

Mathematisches Institut, Universität Bonn, 53115 Bonn, Germany

E-mail address: Daniel@Wienholtz.de

\section{REFERENCES}

[AA] W. K. Allard and F. J. Almgren, Jr., The structure of stationary one dimensional varifolds with positive density, Invent. Math. 34 (1976), 83-97.

[All1] W. K. Allard, On the first variation of a varifold, Ann. of Math. 95 (1972), $417-491$.

[All2] On the first variation of a varifold: boundary behavior, Ann. of Math. 101 (1975), 418-446.

[Alt] H. W. Alt, Verzweigungspunkte von H-Flächen, I, Math. Z. 127 (1972), 333-362; II, Math. Ann. 201 (1973), 33-55.

[AT] F. J. Almgren, JR. and W. P. Thurston, Examples of unknotted curves which bound only surfaces of high genus within their convex hull, Ann. of Math. 105 (1977), 527-538.

[AS] F. J. Almgren, JR. and L. Simon, Existence of embedded solutions of Plateau's problem, Ann. Scuola Norm. Sup. Pisa 6 (1979), 447-495.

[Be] F. Bernatzki, The Plateau-Douglas problem for nonorientable minimal surfaces, Manuscripta Math. 79 (1993), 73-80.

[Boh] R. Bӧнме, New results on the classical problem of Plateau. On the existence of many solutions, Séminaire Bourbaki, Astérisque 92 (1981/82), 1-20. 
[Bor] K. Bonsuk, Sur la courbure totale des courbes fermées, Annales Soc. Polonaise math. 20 (1947), 251-265.

[DHKW] U. Dierkes, S. Hildebrandt, A. Küster, and O. Wohlrab, Minimal Surfaces, Vols. I \& II, Springer-Verlag, New York, 1992.

[DW] J. Drachman and B. White, Soap films bounded by non-closed curves, J. Geom. Anal. 8 (1998), 239-250.

[Fa] M. I. FÁry, Sur la courbure totale d'une courbe gauche faisant un nœud, Bull. Soc. Math. France 77 (1949), 128-138.

[Fed] H. Federer, Some theorems on integral currents, Trans. Amer. Math. Soc. 117 (1965), 43-67.

[Fen] W. Fenchel, Über Krümmung und Windung geschlossener Raumkurven, Math. Ann. 101 (1929), 238-252.

[Gr] M. Gromov, Filling Riemannian manifolds, J. Differential Geom. 18 (1983), 1147.

[Gu1] R. Gulliver, Regularity of minimizing surfaces of prescribed mean curvature, Ann. of Math. 97 (1973), 275-305.

[Gu2] A minimal surface with an atypical boundary branch point, in Differential Geometry, Pitman Surveys Pure Appl. Math. 52 (H. B. Lawson, Jr. and K. Tenenblat, eds.) Proc. of conference for M. do-Carmo, Longman/Wiley, Harlow/New York, 1991, 211-228.

[GuL] R. Gulliver and F. LESLEy, On boundary branch points of minimizing surfaces, Arch. Rat. Mech. Anal. 52 (1973), 20-25.

[GuS] R. Gulliver and J. Spruck, On embedded minimal surfaces, Ann. of Math. 103 (1976), 331-347; correction Ann. of Math. 109 (1979), 407-412.

[H] S. Hildebrandt, Boundary behavior of minimal surfaces, Arch. Rat. Mech. Anal. 35 (1969), 47-82.

[HS] R. HARDT and L. Simon, Boundary regularity and embedded solutions for the oriented Plateau problem, Ann. of Math. 110 (1979), 439-486.

[HT] E. Heinz and F. Tomi, Zu einem Satz von Hildebrandt über das Randverhalten von Minimalflächen, Math. Z. 111 (1969), 372-386.

[Hub] J. H. Hubbard, On the convex hull genus of space curves, Topology 19 (1980), 203-208.

[J] J. Jost, Conformal mappings and the Plateau-Douglas problem in Riemannian manifolds, J. Reine Angew. Math. 359 (1985), 37-54.

[K] D. Kinderlehrer, The boundary regularity of minimal surfaces, Ann. Scuola Norm. Sup. Pisa 23 (1969), 711-744.

$[\mathrm{Ku}] \quad$ N. KuIPER, Geometry in curvature theory, in Tight and Taut Submanifolds (T. E. Cecil and S.-s. Chern, eds.), Math. Sci. Res. Inst. Publication 32, Cambridge Univ. Press, Cambridge, 1997, 1-50.

[L] F. D. LeSley, Differentiability of minimal surfaces at the boundary, Pacific J. Math. 37 (1971), 123-139.

[Me] W. H. Meeks, III, Uniqueness theorems for minimal surfaces, Illinois J. Math. 25 (1981), 318-336.

[Mi] J. Milnor, On the total curvature of knots, Ann. of Math. 52 (1950), 248-257.

[Mo1] F. Morgan, A regularity theorem for minimizing hypersurfaces modulo $\nu$, Trans. Amer. Math. Soc. 297 (1986), 243-253.

[Mo2] Geometric Measure Theory: A Beginner's Guide, Academic Press, San Diego, CA, 1995.

[MW] W. H. Meeks, III and B. White, The space of minimal annuli bounded by an extremal pair of planar curves, Comm. Anal. Geom. 1 (1993), 415-437. 
[MY1] W. H. Meers, III and S. T. Yau, The classical Plateau problem and the topology of three-dimensional manifolds. The embedding of the solution given by DouglasMorrey and an analytic proof of Dehn's lemma, Topology 21 (1982), 409-442.

[MY2] W. H. MeEks, III and S. T. YAu, The existence of embedded minimal surfaces and the problem of uniqueness, Math. Z. 179 (1982), 151-168.

[N1] J. C. C. Nitsche, MR 25 \#492, (review of Sasaki's paper [Sa]), Math. Rev. 25 (1963), 104.

[N2] The boundary behavior of minimal surfaces. Kellog's theorem and Branch points on the boundary, Invent. Math. 8 (1969), 313-333; Concerning my paper on the boundary behavior of minimal surfaces, Invent. Math. 9 (1969/70), 270.

[N3] A new uniqueness theorem for minimal surfaces, Arch. Rat. Mech. Anal. 52 (1973), 319-329.

[N4] Lectures on Minimal Surfaces, Vol. I, Cambridge Univ. Press, Cambridge, 1989.

[O1] R. Osserman, A proof of the regularity everywhere of the classical solution to Plateau's problem, Ann. of Math. 91 (1970), 550-569.

[O2] On Bers' theorem on isolated singularities, Indiana Univ. Math. J. 23 (1973), 337-342.

[OS] R. Osserman and M. Schiffer, Doubly-connected minimal surfaces, Arch. Rational Mech. Anal. 58 (1975), 285-307.

[PR] J. Pitts and H. Rubinstein, Existence of minimal surfaces of bounded topological type in three-manifolds, in Proc. Centre Math. Anal. Austral. Nat. Univ., Miniconference on geometry and partial differential equations (Canberra, 1985), Vol. 10, Canberra, 1986, 163-176.

[Ra1] T. Radó, On Plateau's problem Ann. of Math. 31 (1930), 457-469.

[Ra2] On the Problem of Plateau, Springer-Verlag, New York, 1971.

[Sa] S. SASAKI, On the total curvature of a closed curve, Japanese J. Math. 29 (1959), $118-125$.

[Sch] R. Schneider, A note on branch points of minimal surfaces, Proc. Amer. Math. Soc. 17 (1966), 1254-1257.

[Sh1] M. Shiffman, The Plateau problem for minimal surfaces of arbitrary topological structure, Amer. J. Math. 61 (1939), 853-882.

[Sh2] Unstable minimal surfaces with several boundaries, Ann. of Math. 43 (1942), 197-222.

[Si] L. Simon, Lectures on Geometric Measure Theory, Proc. of the Centre for Math. Anal. 3, Australian National University Centre for Mathematical Analysis, Canberra, Australia, 1983, vii+272pp.

[Sm] S. Smale, An infinite dimensional version of Sard's theorem, Amer. J. Math. 87 (1965), 861-866.

[TT1] F. Tomi and A. J. Tromba, Extreme curves bound embedded minimal surfaces of the type of the disc, Math. Z. 158 (1978), 137-145.

[TT2] Existence theorems for minimal surfaces of nonzero genus spanning a contour, Mem. Amer. Math. Soc. 71 (1988), iv+83 pp.

[TT3] The index theorem for minimal surfaces of higher genus, Mem. Amer. Math. Soc. 117 (1995), vi+78 pp.

[War] S. E. Warschawski, Boundary derivatives of minimal surfaces, Arch. Rational Mech. Anal. 38 (1970), 241-256.

[Wh1] B. White, A regularity theorem for minimizing hypersurfaces mod $p$, in Geometric Measure Theory and the Calculus of Variations, Proc. Sympos. Pure Math. 44 (1986), Amer. Math. Soc., 413-427.

[Wh2] The space of $m$-dimensional surfaces that are stationary for a parametric elliptic functional, Indiana Univ. Math. J. 36 (1987), 567-602. 
[Wh3] B. White, Curvature estimates and compactness theorems in 3-manifolds for surfaces that are stationary for parametric elliptic functionals, Invent. Math. 88 (1987), 243-256.

[Wh4] B. White, Half of Enneper's surface minimizes area, in Geometric Analysis and the Calculus of Variations for Stefan Hildebrandt (J. Jost, ed.), International Press, Cambridge, MA, 1996, 361-367.

[Wh5] Classical area minimizing surfaces with real-analytic boundaries, Acta Math. 179 (1997), 295-305.

[Wi] D. Wienholtz, A method to exclude branch points of minimal surfaces, Calc. Var. Partial Differential Equations 7 (1998), 219-247.

(Received September 15, 2000) 